\title{
Necessary Optimality Conditions for Determining of the Position of the Boundary of Oil Deposit
}

\author{
N.K. Shazhdekeyeva, B. Kenzhegulov, A.N. Myrzasheva, G.T. Kabylkhamitov, R.U. Tuleuova \\ Department of Mathematics and Methods of Teaching Mathematics, Atyrau State University named after Kh.Dosmukhamedov, Student Ave., \\ 1 -060011, Atyrau, Republic of Kazakhstan.
}

\begin{abstract}
:
The essential criteria was discovered in the thesis to define free boundary over the nonlinear partial differential equations. The task is to find co-ordinates of each point of the border so that to minimize functional. Such task can be treated as an optimum control problem. It is a problem with restriction. In accordance with a method of the interfaced functions we will pass to a problem of optimization without restrictions, having entered new functional. The purpose of the given work is calculation of the first variation of functional. It also defines a necessary condition of optimality for definition of position of border of an oil layer. The considered method for determining free boundaries is based on solving problems of determining the boundary of a system that describes a nonlinear partial differential equation.
\end{abstract}

Keywords - Differential Equation, Functional, Dirac Function, Ecessary Optimality, Initial and Boundary Conditions, Gradient, Laplacian of the Functions, Variation, Integration.

\section{INTRODUCTION}

The task of the current study is to find co-ordinates of each point of the border of the oil deposit so that to minimize functional. Such task can be treated as an optimum control problem. It is a problem with restriction.

The purpose of the given work is calculation of the first variation of functional.

This subsection considers the necessary optimality condition for determining of the position of the boundary of the oil deposit.

Let us consider the problem of determining of the boundary for a system described by nonlinear differential equations in partial derivatives.

$$
u_{t}=f\left(x, y, t, u, u_{x}, u_{y}, u_{x x}, u_{y y}\right)
$$

where $u(x, y, t)-m$-dimensional a vector function of the state of the system, defined in a two-dimensional region $\sigma \in E^{2} ; \quad u_{t}, u_{x}, u_{x x}$ and so on - denoting the partial derivatives with respect to time and spatial coordinates, respectively.

The initial and boundary conditions are given in general form

$$
u(x, y, 0)=u_{0}(x, y) \quad(x, y) \in \sigma
$$

$$
g\left(t, x, y, u, u_{n}\right)=0 \quad(x, y) \in \partial \sigma
$$

where $u_{n}$ denotes the normal derivative of the vector $u$ to the boundary of the region $\partial \sigma$.

\section{MATERIAL AND METHODS}

In accordance with a method of the interfaced functions it is supposed to pass to a problem of optimization without restrictions, having entered new functional.

The considered method for determining free boundaries is based on solving problems of determining the boundary of a system that describes a nonlinear partial differential equation. Principles for solving ill-defined problems are considered in the works of Zakirov and Lapuk [1], Lions [2], Bubnov [3], Bulygin [4-5], Gutnikov et al. [6-8].

Applied to the inverse problems of the filtration theory in the works of Zhirov [9], Shazhdekeeva and Mukhambetzhanov [10], Shazhdekeeva et al. [11], Frolov [12].

\section{RESULTS AND DISCUSSION}

The system evolves over a period of time $t \in[0, T]$, during which the measurements are made.

Observations are represented by the q-dimensional $(\mathrm{q}<\mathrm{m})$ vector $\mathrm{z}(\mathrm{x}, \mathrm{y}, \mathrm{t})$, which for convenience can be considered a continuous function $z \in E^{q}(\sigma \times[0, T])$. Suppose that the vector $\mathrm{z}$ is related to the state of the system by the relation

$$
z(x, y, t)=h(u)+\xi(x, y, t),
$$

where $\xi(x, y, t)-$ measurement error. The problem is to determine the region $\sigma$ (i.e., finding the coordinates of each boundary point $\partial \sigma$ ) in such a way as to minimize the functional

$J=\int_{0}^{T} \iint_{\sigma \sigma}[z(x, y, t)-h(u(x, y, t))]^{\prime} G(x, y, \xi, \eta, t) \times[z(\xi, \eta, t)-h(u(\xi, \eta, t))] d x d y d \xi d \eta d t$

The weight matrix $G(x, y, \xi, \eta, t)$ is continuous in its arguments, positive definite and symmetric ( $G=G^{\prime}$ ).In this case, the problem under consideration can be treated as an optimal control problem, in which the position of the boundary is a control variable. 
International Journal of Engineering Research and Technology. ISSN 0974-3154, Volume 13, Number 6 (2020), pp. 1204-1209

(C) International Research Publication House. https://dx.doi.org/10.37624/IJERT/13.6.2020.1204-1209

In practice, the case of measuring the state vector of a system in M-discrete points $\left(x_{j}, y_{j}\right)$ is often encountered, i.e. a case

$$
z\left(x_{j}, y_{j}, t\right)=A_{j} u\left(x_{j}, y_{j}, t\right)+\zeta_{j}(t), j=1,2, \ldots, M
$$

where $A_{j}(j=1,2, \ldots, M)-$ matrices of dimension $(q \times m)$,whose elements are equal to zero or one. In this case, the original functional $\mathrm{J}$ can be represented as follows:

$J=\int_{0}^{T} \sum_{j=1}^{M}\left[z\left(x_{j}, y_{j}, t\right)-A_{j} u\left(x_{j}, y_{j}, t\right)\right]^{\prime} G_{j}(t)\left[z\left(x_{j}, y_{j}, t\right)-A_{j} u\left(x_{j}, y_{j}, t\right)\right] d t$

The notation (1.5.7) can be reduced to a more general form (5) by introducing into the weight function $\sigma$ - a Dirac function. Therefore, in the sequel, for convenience, a more general formula (5) is used.

The optimal control problem formulated above is a problem with the constraint imposed by equation (1.5.1). In accordance with the method of conjugate functions, pass to the optimization problem without restrictions, introducing a new functional

$$
J_{\psi}=J+\int_{0}^{T} \int_{\sigma} \psi^{\prime}(x, y, t)\left[f-u_{t}\right] d x d y d t
$$

where $\psi(x, y, t)-m$-dimensional conjugate vector-valued function. If $\partial \sigma$-boundary, minimizing $J$, then it also minimizes and $J_{\psi}$.

Suppose that the boundary $\partial \sigma$ is subjected to a perturbance and transforms $d \sigma^{*}=d \sigma+\delta(d \sigma)$ with the corresponding transformation of the region $\sigma$ in $\sigma^{*}=\sigma+\delta \sigma$. Perturbance of the border $\partial \sigma$ leads to perturbance $J_{\psi}$. Our goal is to calculate the first variation $J_{\psi}$.

Suppose that a new area $\sigma^{*}$ (its coordinates are indicated $x^{*}, y^{*}$ ), which depends on the parameter $\varepsilon$, can be converted to the original region $\sigma$ by transformations

$$
\begin{aligned}
& x^{*}=\Phi_{1}\left(x, y, u, \nabla u, \nabla^{2} u ; \varepsilon\right) ; \\
& y^{*}=\Phi_{2}\left(x, y, u, \nabla u, \nabla^{2} u ; \varepsilon\right),
\end{aligned}
$$

where $\nabla u$ and $\nabla^{2} u$ gradient, and Laplacian of the functions $u$. The new value of the function $u^{*}=u^{*}\left(x^{*}, y^{*}\right)$ is reduced to the original function $u(x, y)$ by the transformations (9), (10) and the transformation

$$
u^{*}=\Phi_{3}\left(x, y, u, \nabla u, \nabla^{2} u ; \varepsilon\right)
$$

It is assumed that these transformations are continuous, invertible, differentiable, and that to the values $\varepsilon=0$ correspond identical transformations

$$
\begin{aligned}
& x=\Phi_{1}\left(x, y, u, \nabla u, \nabla^{2} u ; 0\right) \\
& y=\Phi_{1}\left(x, y, u, \nabla u, \nabla^{2} u ; 0\right) \\
& u=\Phi_{3}\left(x, y, u, \nabla u, \nabla^{2} u ; 0\right)
\end{aligned}
$$

If $\varepsilon$ - a is small value, then equation (9) - (11) can be represented in the form

$$
\begin{gathered}
x^{*}=\left.\Phi_{1}\right|_{\varepsilon=0}+\left.\varepsilon \frac{\partial \Phi_{1}}{\partial \varepsilon}\right|_{\varepsilon=0}+O(\varepsilon)=x+\varepsilon \varphi_{1}+O(\varepsilon) \\
y^{*}=\left.\Phi_{2}\right|_{\varepsilon=0}+\left.\varepsilon \frac{\partial \Phi_{2}}{\partial \varepsilon}\right|_{\varepsilon=0}+0(\varepsilon)=y+\varepsilon \varphi_{2}+0(\varepsilon) \\
u^{*}=\left.\Phi_{3}\right|_{\varepsilon=0}+\left.\varepsilon \frac{\partial \Phi_{3}}{\partial \varepsilon}\right|_{\varepsilon=0}+0(\varepsilon)=y+\varepsilon \varphi_{3}+O(\varepsilon)
\end{gathered}
$$

The first variations $x, y, u$ are defined as follows:

$$
\begin{gathered}
\delta x=x^{*}-x=\varepsilon \varphi_{1} \\
\delta y=y^{*}-y=\varepsilon \varphi_{2} \\
\delta u=u^{*}\left(x^{*}, y^{*}\right)-u(x, y)=\varepsilon \varphi_{3}
\end{gathered}
$$

The first variation $J_{\psi}$ due to the perturbance is the principal linear part of (relative to $\varepsilon$ ) of the difference:

$J_{\psi}\left[u^{*}\left(x^{*}, y^{*}\right)\right]-J_{\psi}[u(x, y)]=\int_{0}^{T} \int_{\sigma^{*} \sigma^{*}}^{T} \int\left[z-h\left(u^{*}\right)\right]^{\prime} \times G\left(x^{*}, y^{*}, \xi^{*}, \eta^{*}, t\right)\left[z-h\left(u^{*}\right)\right] d x^{*} d y^{*} d \xi^{*} d \eta^{*} d t-$

$-\int_{0}^{T} \int_{\sigma \sigma} \int_{\sigma}[z-h(u)]^{\prime} G(x, y, \xi, \eta, t)[z-h(u)] \times d x d y d \xi d \eta d t+\int_{0}^{T} \int_{\sigma^{*}} \psi^{\prime}\left[f-u_{t}^{*}\right] d x^{*} d y^{*} d t-$

$$
-\int_{0}^{T} \int_{\sigma} \psi^{\prime}\left[f-u_{t}\right] d x d y d t
$$

Using the Jacobian of the transformation, we reduce equation (21) to the form

$$
\begin{gathered}
J_{\psi}\left[u^{*}\right]-J_{\psi}[u]=\int_{0}^{T} \int_{\sigma \sigma} \int_{\sigma}\left[z-h\left(u^{*}\right)\right]^{\prime} G\left[z-h\left(u^{*}\right)\right] \times\left|\frac{\partial\left(x^{*}, y^{*}, \xi^{*}, \eta^{*}\right)}{\partial(x, y, \xi, \eta)}\right| d x d y d \xi d \eta d t- \\
-\int_{0}^{T} \int_{\sigma \sigma} \int_{\sigma}[z-h(u)]^{\prime} G \times[z-h(u)] d x d y d \xi d \eta d t+\int_{0}^{T} \int_{\sigma} \psi^{\prime}\left[f-u_{t}^{*}\right] \times\left|\frac{\partial\left(x^{*}, y^{*}\right)}{\partial(x, y)}\right| d x d y d t- \\
-\int_{0}^{T} \int_{\sigma} \psi^{\prime}\left[f-u_{t}\right] d x d y d t
\end{gathered}
$$


International Journal of Engineering Research and Technology. ISSN 0974-3154, Volume 13, Number 6 (2020), pp. 1204-1209

(C) International Research Publication House. https://dx.doi.org/10.37624/IJERT/13.6.2020.1204-1209

where

$\left|\frac{\partial\left(x^{*}, y^{*}, \xi^{*}, \eta^{*}\right)}{\partial(x, y, \xi, \eta)}\right| \approx 1+\varepsilon \frac{\partial \varphi_{1}}{\partial x}+\varepsilon \frac{\partial \varphi_{2}}{\partial y}+\varepsilon \frac{\partial \varphi_{1}}{\partial \xi}+\varepsilon \frac{\partial \varphi_{2}}{\partial \eta}$,

$\left|\frac{\partial\left(x^{*}, y^{*}\right)}{\partial(x, z)}\right| \approx 1+\varepsilon \frac{\partial \varphi_{1}}{\partial x}+\varepsilon \frac{\partial \varphi_{2}}{\partial y}$.

Expanding the integrals in the expression (22) in a Taylor series and preserving terms of the first order with respect to $\varepsilon$, obtain the first variation $J_{\psi}$ in the form

$\delta J_{\psi}=-\int_{0}^{T} \int_{\sigma \sigma} \int_{\sigma} 2[z(\xi, \eta, t)-h(u(\xi, \eta, t))]^{\prime} G^{\prime}(x, y, \xi, \eta, t) \times h_{u}(u(x, y, t))\left[\delta \bar{u}+u_{x} \delta x+\right.$

$\left.+u_{y} \delta y\right] d x d y d \xi d \eta d t++\int_{0}^{T} \int_{\sigma} \int_{\sigma} 2[z(x, y, t)-h(u(x, y, t))]^{\prime} G(x, y, \xi, \eta, t) \times$

$\times[z(\xi, \eta, t)-h(u(\xi, \eta, t))]\left[\frac{\partial \delta x}{\partial x}+\frac{\partial \delta y}{\partial y}\right] d x d y d \xi d \eta d t++\int_{0}^{T} \int_{\sigma}\left[\psi^{\prime}\left(f-u_{t}\right)\left(\frac{\partial \delta x}{\partial x}+\frac{\partial \delta y}{\partial y}\right)+\right.$

$+\delta \psi^{\prime}\left(f-u_{t}\right)+\psi^{\prime} f_{x} \delta x+\psi^{\prime} f_{y} \delta y+\psi^{\prime} f_{u} \delta \bar{u}+\psi^{\prime} f_{u} u_{x} \delta x+\psi^{\prime} f_{u} u_{y} \delta y+\psi^{\prime} f_{u_{x}} \overline{\delta u_{x}}+$

$+\psi^{\prime} f_{u_{x}} u_{x y} \delta y+\psi^{\prime} f_{u_{y}} \overline{\delta u_{y}}+\psi^{\prime} f_{u_{y}} u_{y x} \delta x+\psi^{\prime} f_{u_{y}} u_{y y} \delta y+\psi^{\prime} f_{u_{x x}} \overline{\delta u_{x x}}+\psi^{\prime} f_{u_{x x}} u_{x x x} \delta x+\psi^{\prime} f_{u_{x}} u_{x x} \delta x+$

$\left.+\psi^{\prime} f_{u_{x}} u_{x x y} \delta y+\psi^{\prime} f_{u_{y y}} \overline{\delta u_{y y}}+\psi^{\prime} f_{u_{y y}} u_{y y x} \delta x+\psi^{\prime} f_{u_{y y}} u_{y y y} \delta y-\psi^{\prime} \overline{\delta u_{t}}-\psi^{\prime} u_{t x} \delta x-\psi^{\prime} u_{t y} \delta y\right] d x d y d t$,

there

$\delta \psi=\psi\left(x^{*}, y^{*}, t\right)-\psi(x, y, t)=\psi_{x} \delta x+\psi_{y} \delta y, \overline{\delta u}=u^{*}(x, y, t)-u(x, y, t), \overline{\psi^{\prime}} \overline{\delta u_{t}}=\frac{\partial}{\partial t}\left(\psi^{\prime} \overline{\delta u}\right)-\frac{\partial \psi^{\prime}}{\partial t} \overline{\delta u}$
The following relations are also used:

$$
+\psi^{\prime} f_{u_{x x}} u_{x x y} \delta y+\psi^{\prime} f_{u_{y y}} u_{y y y} \delta y
$$

$\frac{\partial}{\partial x}\left(\psi^{\prime} u_{t} \delta x\right)+\frac{\partial}{\partial y}\left(\psi^{\prime} u_{t} \delta y\right)=\frac{\partial \psi^{\prime}}{\partial x} u_{t} \delta x+\psi^{\prime} u_{t x} \delta x+\psi^{\prime} u_{t} \delta x_{x}+\frac{\partial \psi^{\prime}}{\partial y} u_{t} \delta y+\psi^{\prime} u_{t y} \delta y+\psi^{\prime} u_{t} \delta y_{y} ;$ $\psi^{\prime} f_{u_{x}} \overline{\delta u_{x}}+\psi^{\prime} f_{u_{y}} \overline{\delta u_{y}}=\frac{\partial}{\partial x}\left(\psi^{\prime} f_{u_{x}} \overline{\delta u}\right)-\frac{\partial}{\partial x}\left(\psi^{\prime} f_{u_{x}}\right) \overline{\delta u}+\frac{\partial}{\partial y}\left(\psi^{\prime} f_{u_{y}} \overline{\delta u}\right)-\frac{\partial}{\partial y}\left(\psi^{\prime} f_{u_{y}}\right) \overline{\delta u} ;$

$$
\begin{gathered}
\psi^{\prime} f_{u_{x x}} \overline{\delta u_{x x}}+\psi^{\prime} f_{u_{y y}} \overline{\delta u_{y y}}=-\frac{\partial}{\partial x}\left[\left(\psi^{\prime} f_{u_{x x}}\right)_{x} \overline{\delta u}\right]+\frac{\partial^{2}}{\partial x^{2}}\left(\psi^{\prime} f_{u_{x x}}\right) \overline{\delta u}+\frac{\partial}{\partial x}\left(\psi^{\prime} f_{u_{x x}} \overline{\delta u_{x}}\right)-\frac{\partial}{\partial y}\left[\left(\psi^{\prime} f_{u_{y y}}\right)_{y} \overline{\delta u}\right]+ \\
+\frac{\partial^{2}}{\partial y^{2}}\left(\psi^{\prime} f_{u_{y y}}\right) \overline{\delta u}+\frac{\partial}{\partial y}\left(\psi^{\prime} f_{u_{y y}} \overline{\delta u_{y}}\right)
\end{gathered}
$$

and the relation $\overline{\delta u}(x, y, 0)=0$, obtain the first variation of the functional $\delta J_{\psi}$ in the form

$$
\begin{gathered}
\delta J_{\psi}=\int_{0}^{T} \int_{\sigma}\left\{\frac{\partial \psi^{\prime}}{\partial t}+\psi^{\prime} f_{u}-\frac{\partial}{\partial x}\left(\psi^{\prime} f_{u_{x}}\right)-\frac{\partial}{\partial y}\left(\psi^{\prime} f_{u_{y}}\right)+\frac{\partial^{2}}{\partial x^{2}}\left(\psi^{\prime} f_{u_{x x}}\right)+\frac{\partial^{2}}{\partial y^{2}}\left(\psi^{\prime} f_{u_{y y}}\right)-2 \int_{\sigma}[z(\xi, \eta, t)-\right. \\
\left.-h(u(\xi, \eta, t))]^{\prime} G^{\prime}(x, y, \xi, \eta, t) h_{u}(u(x, y, t)) d \xi d \eta\right\} \times \overline{\delta u} d x d y d t+\int_{0}^{T} \int_{\sigma}\left\{\frac { \partial } { \partial x } \left[\psi^{\prime} f_{u_{x}} \overline{\delta u}-\left(\psi^{\prime} f_{u_{x x}}\right)_{x} \overline{\delta u}+\right.\right. \\
\left.+\psi f_{u_{x x}} \overline{\delta u_{x}}+\left(\int_{\sigma}[z(x, y, t)-h(u(x, y, t))]^{\prime} \times G(x, y, \xi, \eta, t)[z(\xi, \eta, t)-h(u(\xi, \eta, t))] d \xi d \eta\right) \delta x\right]+ \\
+\frac{\partial}{\partial y}\left[\psi^{\prime} f_{u_{y}} \overline{\delta u}-\left(\psi^{\prime} f_{u_{y y}}\right)_{y} \overline{\delta u}+\psi^{\prime} f_{u_{y y}} \delta u_{y}+2\left(\int_{\sigma}[z(x, y, t)-h(u(x, y, t))]^{\prime} G(x, y, \xi, \eta, t) \times[z(\xi, \eta, t)-\right.\right.
\end{gathered}
$$


International Journal of Engineering Research and Technology. ISSN 0974-3154, Volume 13, Number 6 (2020), pp. 1204-1209

(C) International Research Publication House. https://dx.doi.org/10.37624/IJERT/13.6.2020.1204-1209

$$
-h(u(\xi, \eta, t))] d \xi d \eta) \delta y]\} d x d y d t-\left.\int_{\sigma} \psi^{\prime} \overline{\delta u}\right|_{t=T} d x d y .
$$

Let the conjugate vector $\psi(x, y, t)$ satisfy the system of equations

$$
\delta J_{\psi}=\int_{0}^{T} \int_{\partial \sigma}\left\{\left[\psi^{\prime} f_{u_{x}}-\left(\psi^{\prime} f_{u_{x x}}\right)_{x}\right] \cos v+\left[\psi^{\prime} f_{u_{y}}-\left(\psi^{\prime} f_{u_{y y}}\right)_{y}\right] \sin v+\right.
$$

$$
\begin{aligned}
\psi_{t}=-f_{u}^{\prime} \psi+\left(f_{u_{x}}^{\prime} \psi\right)_{x}+\left(f_{u_{y}}^{\prime} \psi\right)_{y}-\left(f_{u x x}^{\prime} \psi\right)_{x x}-\left(f_{u_{y y}}^{\prime} \psi\right)_{y y}+2 \int_{\sigma} h_{u}^{\prime}(x, y, t) G(x, y, \xi, \eta, t) \times & \\
& \left.+\frac{\partial}{\partial x}\left[\psi^{\prime} f_{u_{x x}} \sin v \cos v-\psi^{\prime} f_{u_{y y}} \cos v \sin v\right]\right\} \delta u d s d t+
\end{aligned}
$$

$$
\times[z(\xi, \eta, t)-h(u(\xi, \eta, t))] d \xi d \eta
$$

with the condition at the end of the time interval

$$
\psi(x, y, T)=0 .
$$

The substitution of the equations (30) and (31) into the expression (29) and the application of the Gauss-Ostrogradskii theorem gives the following representation for the variation of the functional:

$\delta J_{\psi}=\int_{0}^{T} \int\left\{\left[\psi^{\prime} f_{u_{x}}-\left(\psi^{\prime} f_{u_{x x}}\right)_{x}\right] \cos v+\left[\psi^{\prime} f_{u_{y}}-\left(\psi^{\prime} f_{u_{y y}}\right)_{y}\right] \sin v\right\} \overline{\delta u} d s d t+$

$+\int_{0}^{T} \int_{\partial \sigma}\left(\psi^{\prime} f_{u_{x x}} \overline{\delta u_{x}} \cos v+\psi^{\prime} f_{u_{y y}} \overline{\delta u_{y}} \sin v\right) d s d t+\int_{0}^{T} \int_{\partial \sigma}\left\{2 \int_{\sigma}[z(x, y, t)-h(u(x, y, t))]^{\prime} G(x, y, \xi, \eta, t) \times\right.$

$$
\times[z(\xi, \eta, t)-h(u(\xi, \eta, t))] d \xi d \eta\}[\delta x \cos v+\delta y \sin v] d s d t,
$$

where $v$-the angle between the positive direction of the $x$ axis and the outer normal to the boundary of the region $\partial \sigma$, $s$ - the arc length of the contour $\partial \sigma$.

To determine the boundary conditions for a function $\psi$ it is more convenient to use a $\delta u$. Expressing $\overline{\delta u}$ through $\delta u$ and substituting this expression in (32), obtain

$$
\begin{array}{r}
\delta J_{\psi}=\int_{0}^{T} \int_{\partial \sigma}\left\{\left[\psi^{\prime} f_{u_{x}}-\left(\psi^{\prime} f_{u_{x x}}\right)_{x}\right] \cos v+\left[\psi^{\prime} f_{u_{y}}-\left(\psi^{\prime} f_{u_{y y}}\right)_{y}\right] \sin v\right\} \delta u d s d t+ \\
+\int_{0}^{T} \int_{\partial \sigma}\left(\psi f_{u_{x x}}-\overline{\delta u_{x}} \cos v+\psi f_{u_{y y}} \overline{\delta u_{y}} \sin v\right) d s d t+
\end{array}
$$$$
+\int_{0}^{T} \int_{\partial \sigma}\left\{S(x, y, t,)(\delta x \cos v+\delta y \sin v)-\left[\left(\psi^{\prime} f_{u_{x}}-\left(\psi^{\prime} f_{u_{x x}}\right)_{x}\right) \cos v+\right.\right.
$$$$
\left.\left.+\left(\psi^{\prime} f_{u_{y}}-\left(\psi^{\prime} f_{u_{y y}}\right)_{y}\right) \sin v\right]\left(u_{x} \delta x+u_{y} \delta y\right)\right\} d s d t
$$

where is denoted

$S(x, y, t)=,2 \int_{\sigma}[z(x, y, t)-h(u(x, y, t))]^{\prime} G(x, y, \xi, \eta, t)[z(\xi, \eta, t)-h(u(\xi, \eta, t))] d \xi d \eta$

If we express both $\overline{\delta u_{x}}$ and $\overline{\delta u_{y}}$ in terms of the normal $\overline{\delta u_{n}}$ and tangential $\overline{\delta u_{s}}$ derivatives of the function $u$ on $\partial \sigma$, and also assume that the contour $\partial \sigma$ is closed, then equation (1.5.33) is transformed to the form

$$
\left.+\psi^{\prime} f_{u_{y y}} \sin ^{2} v\right) \overline{\delta u_{n}} d s d t+\int_{0}^{T} \int_{\partial \sigma}\left\{S(x, y, t)(\delta x \cos v+\delta y \sin v)-\left[\left(\psi^{\prime} f_{u_{x}}-\left(\psi^{\prime} f_{u_{x x}}\right)_{x}\right) \cos v+\right.\right.
$$$$
+
$$$$
\left(\psi^{\prime} f_{u_{y}}-\left(\psi^{\prime} f_{u_{y y}}\right)_{y} \sin v\right]\left[u_{x} \delta x+u_{y} \delta y\right]-\frac{\partial}{\partial s}\left(\psi^{\prime} f_{u_{x x}} \sin v \cos v-\right.
$$

$$
\left.\left.-\psi^{\prime} f_{u_{y y}} \sin v \cos v\right)\left(u_{x} \delta x+u_{y} \delta y\right)\right\} d s d t
$$

For convenience of calculations, the variation $\bar{\delta}_{n}=\partial u^{*}(x, y) / \partial n-\partial u(x, y) / \partial n$ can be expressed in terms of $\delta u_{n}=\partial u^{*}\left(x^{*}, y^{*}\right) / \partial n^{*}-\partial u(x, y) / \partial n$.

Indeed, since the angle between $n$ and $n^{*}$, obtain $\frac{\partial u^{*}(x, y)}{\partial n}-\frac{\partial u(x, y)}{\partial n}=\frac{\partial u^{*}\left(x^{*}, y^{*}\right)}{\partial n^{*}}-\frac{\partial u(x, y)}{\partial n}-\frac{\partial^{2} u(x, y)}{\partial n^{2}} \delta n-\frac{\partial^{2} u(x, y)}{\partial n \partial s} \delta s$,

where $n$ - normal direction to $\partial \delta$ at the point $(x, y) ; s$ - the tangent direction to $\partial \delta$ at the point $(\mathrm{x}, \mathrm{y}) ; n^{*}-$ the normal direction to the perturbed boundary $\partial \sigma^{*}$ at the point $\left(x^{*}, y^{*}\right.$ ) , which after deformation corresponds to the point $(x, y)$ on $\partial \delta ; \partial n$ and $\partial s-$ are the normal and tangential components of the variations $\delta x$ and $\delta y$.

The substitution of (35) into (34) gives

$\delta J_{\psi}=\int_{0}^{T} \int_{\partial \sigma}\left\{\left[\psi^{\prime} f_{u_{x}}-\left(\psi^{\prime} f_{u_{x x}}\right)_{x}\right] \cos v+\left[\psi^{\prime} f_{u_{y}}-\left(\psi^{\prime} f_{u_{y y}}\right)_{y}\right] \sin v+\frac{\partial}{\partial x}\left[\psi^{\prime} f_{u_{x x}} \sin v \cos v-\right.\right.$

$$
\begin{gathered}
+\frac{\partial}{\partial x} \psi^{\prime} f_{u_{x x}} \sin v \cos v- \\
\left.\left.\psi^{\prime} f_{u_{y y}} \sin v \cos v\right]\right\} \delta u d s d t+
\end{gathered}
$$

$+\int_{0}^{T} \int_{\partial \sigma}\left(\psi^{\prime} f_{u_{x x}} \cos ^{2} v+\psi^{\prime} f_{u_{y y}} \sin ^{2} v\right) \delta u_{n} d s d t-\int_{0}^{T} \int_{\partial \sigma}\left(\psi^{\prime} f_{u_{x x}} \cos ^{2} v+\right.$ $\left.+\psi^{\prime} f_{u_{y y}} \sin ^{2} v\right)\left(\frac{\partial^{2} u}{\partial n^{2}} \delta n+\frac{\partial^{2} u}{\partial n \partial s} \delta s\right) d s d t+\int_{0}^{T} \int_{\partial \sigma}\{S(x, y, t)(\delta x \cos v+\delta y \sin v)-$ $-\left[\left(\psi^{\prime} f_{u_{x}}-\left(\psi^{\prime} f_{u_{x x}}\right)_{x} \cos v+\left(\psi^{\prime} f_{u_{y}}-\left(\psi^{\prime} f_{u_{y y}}\right)_{y}\right) \sin v\right)\right]\left[u_{x} \delta x+u_{y} \delta y\right]-$ 
International Journal of Engineering Research and Technology. ISSN 0974-3154, Volume 13, Number 6 (2020), pp. 1204-1209

(C) International Research Publication House. https://dx.doi.org/10.37624/IJERT/13.6.2020.1204-1209

$\left.-\frac{\partial}{\partial s}\left(\psi^{\prime} f_{u_{x x}} \sin v \cos v-\psi^{\prime} f_{u_{y y}} \sin v \cos v\right)\left(u_{x} \delta x+u_{y} \delta y\right)\right\} d s d t$

It follows from (3) that

$$
\begin{gathered}
g_{x} \delta x+g_{y} \delta y+g_{u} \delta u+g_{u_{n}} \delta u_{n}=0 \\
x, y \in \partial \sigma \\
x^{*}, y^{*} \in \partial \sigma^{*}
\end{gathered}
$$

Solving (37) with respect to $\delta u_{n}$ and substituting the result in (36), obtain

$\delta J_{\psi}=\int_{0}^{T} \int_{\delta \sigma \sigma}\left\{\left[\psi^{\prime} f_{u_{x}}-\left(\psi^{\prime} f_{u_{x x}}\right)_{x}\right] \cos v+\left[\psi^{\prime} f_{u_{y}}-\left(\psi^{\prime} f_{u_{y}}\right)_{y}\right] \sin v+\frac{\partial}{\partial s}\left[\psi^{\prime} f_{u_{x}} \sin v \cos v-\right.\right.$

$\left.-\psi^{\prime} f_{u_{y y}} \sin v \cos v-\left(\psi^{\prime} f_{u_{x x}} \cos ^{2} v+\psi^{\prime} f_{u_{y y}} \sin ^{2} v\right) g_{u_{n}}^{-1} g_{u}\right\} \delta u d s d t+$

$$
\int_{0}^{T} \int_{\partial \sigma} \cos v-
$$

$-\left[\left(\psi^{\prime} f_{u_{x}}-\left(\psi^{\prime} f_{u_{x x}}\right)_{x}\right) \cos v+\left(\psi^{\prime} f_{u_{y}}-\left(\psi^{\prime} f_{u_{y y}}\right)_{y}\right) \sin v\right] u_{x}-\frac{\partial}{\partial s}\left(\psi^{\prime} f_{u_{x x}} \sin v \cos v-\right.$

$\left.-\psi^{\prime} f_{u_{y y}} \sin v \cos v\right) u_{x}-\left(\psi^{\prime} f_{u_{x x}} \cos ^{2} v+\psi^{\prime} f_{u_{y y}} \sin ^{2} v g^{-1} u_{n} g_{x}-\left(\psi^{\prime} f_{u_{x x}} \cos ^{2} v+\right.\right.$

$\left.\psi^{\prime} f_{u_{y y}} \sin ^{2} v\left(\frac{\partial^{2} u}{\partial n^{2}} \cos v-\frac{\partial^{2} u}{\partial n \partial s} \sin v\right)\right\} \delta x d s d t+\int_{0}^{T} \int_{\partial \sigma}\{S(x, y, t) \sin v-$

$$
\left[\left(\psi^{\prime} f_{u_{x}}-\left(\psi^{\prime} f_{u_{x x}}\right)_{x}\right) \cos v+\right.
$$

$\left.\left(\psi^{\prime} f_{u_{y}}-\left(\psi^{\prime} f_{u_{y y}}\right)_{y}\right) \sin v\right] u_{y}-\frac{\partial}{\partial s}\left(\psi^{\prime} f_{u_{x x}} \sin v \cos v-\psi^{\prime} f_{u_{y y}} \sin v \cos v\right) u_{y}-\left(\psi^{\prime} f_{u_{x x}} \cos ^{2} v+\right.$

$+$

$\left.\psi^{\prime} f_{u_{y y}} \sin ^{2} v\right) g^{-1}{ }_{u_{n}} g_{y}-\left(\psi^{\prime} f_{u_{x x}} \cos ^{2} v+\psi^{\prime} f_{u_{y y}} \sin ^{2} v\right)\left(\frac{\partial^{2} u}{\partial n^{2}}\right.$

$$
\left.\left.\sin v+\frac{\partial^{2} u}{\partial n \partial s} \cos v\right)\right\} \delta y d s d t
$$

We now choose boundary conditions for equation (30), so that the first term on the right-hand side of expression (38) vanishes. In view of the arbitrariness of the variation $\delta u$ on the contour, obtain

$\left[\psi^{\prime} f_{u_{x}}-\left(\psi^{\prime} f_{u_{x x}}\right)_{x}\right] \cos v+\left[\psi^{\prime} f_{u_{y}}-\left(\psi^{\prime} f_{u_{y y}}\right)_{y}\right] \sin v+\frac{\partial}{\partial s}\left[\psi^{\prime} f_{u_{x x}} \sin v \cos v-\psi^{\prime} f_{u_{y y}} \sin v \cos v\right]-$

$$
-\left(\psi^{\prime} f_{u_{x x}} \cos ^{2} v+\psi^{\prime} f_{u_{y y}} \sin ^{2} v\right) g^{-1}{ }_{u_{n}} g_{u}=0 .
$$

Substituting (30) into (29) and changing the order of integration, obtain the final expression

$$
\delta J_{\psi}=\int_{\partial \sigma} L_{1}(x, y) \delta x(x, y) d s+\int_{\partial \sigma} L_{2}(x, y) \delta y(x, y) d s,
$$

where

$$
\begin{aligned}
& L_{1}(x, y)=\int_{0}^{T}\left\{S(x, y, t) \cos v-\left[\left(\psi^{\prime} f_{u_{x}}-\left(\psi^{\prime} f_{u_{x x}}\right)_{x}\right) \cos v+\left(\psi^{\prime} f_{u_{y}}-\left(\psi^{\prime} f_{u_{y y}}\right)_{y}\right) \sin v\right] u_{x}-\right. \\
& -\frac{\partial}{\partial s}\left(\psi^{\prime} f_{u_{x x}} \sin v \cos v-\psi^{\prime} f_{u_{y y}} \sin v \cos v\right) u_{x}-\left(\psi^{\prime} f_{u_{x x}} \cos ^{2} v+\psi^{\prime} f_{u_{y}} \sin ^{2} v\right) g^{-1} u_{n} g_{x}- \\
& \left.-\left(\psi^{\prime} f_{u_{x x}} \cos ^{2} v+\psi^{\prime} f_{u_{y y}} \sin ^{2} v\right)\left(\frac{\partial^{2} u}{\partial n^{2}} \cos v-\frac{\partial^{2} u}{\partial n \partial s} \sin v\right)\right\} d t, \quad(41) \\
& L_{2}(x, y)=\int_{0}^{T}\left\{S(x, y, t) \sin v-\left[\left(\psi^{\prime} f_{u_{x}}-\left(\psi^{\prime} f_{u_{x x}}\right)_{x}\right) \cos v+\left(\psi^{\prime} f_{u_{y}}-\left(\psi^{\prime} f_{u_{y y}}\right){ }_{y}\right) \sin v\right] u_{y}-\right. \\
& -\frac{\partial}{\partial s}\left(\psi^{\prime} f_{u_{x x}} \sin v \cos v-\psi^{\prime} f_{u_{y y}} \sin v \cos v\right) u_{y}-\left(\psi^{\prime} f_{u_{x x}} \cos ^{2} v+\psi^{\prime} f_{u_{y y}} \sin ^{2} v\right) g^{-1} u_{n} g_{y}-
\end{aligned}
$$
$\left.-\left(\psi^{\prime} f_{u_{x x}} \cos ^{2} v+\psi^{\prime} f_{u_{y y}} \sin ^{2} v\right)\left(\frac{\partial^{2} u}{\partial n^{2}} \sin v+\frac{\partial^{2} u}{\partial n \partial s} \cos v\right)\right\} d t,(x, y) \in \partial \sigma$

Note that the perturbance of the contour $\delta(\partial \sigma)$ is represented by perturbance of the corresponding coordinates $\delta x$ and $\delta y$ contour at each point of the originally defined boundary. The necessary conditions for optimality (of the first order) will be the conditions:

$$
\begin{gathered}
L_{1}(x, y)=0, \\
L_{2}(x, y)=0 .
\end{gathered}
$$

\section{CONCLUSIONS}

The article discovered necessary optimality conditions for determining of the position of the boundary of oil deposit. The essential criteria was discovered in the thesis to define free boundary over the nonlinear partial differential equations.

\section{REFERENCES}

[1] Zakirov SN, Lapuk BB. Design and development of gas fields. Moscow: Nedra, 1974.

[2] Lions J-L. Optimal control of systems described by partial differential equations. Moscow: Mir, 1972.

[3] Bubnov BA. The inverse problem for a parabolic equation and the Cauchy problem for certain classes of evolution equations in classes of functions of finite smoothness. Dokl. AN SSSR, Moscow. 1988;299(5):782-784.

[4] Bulygin VYa. Hydrodynamic analysis of the exploited oil field by solving the correct and ill-posed problems. In: Theory and experiment: problems of development 
of the field. Kazan: Publishing House of Kazan University, 1972.

[5] Bulygin VYa. Hydromechanics of the oil reservoir. Moscow: Nedra, 1974.

[6] Gutnikov AI, Zholdasov A, Zakirov SN. Deposits of gas and oil in natural and artificial streams of water. All-Russian Research Institute of Gas Industry, Seria: Geol. and gas condensate fields, Moscow. 1986;4:54.

[7] Gutnikov AI, Zholdasov A, Zakirov SN. The configurations of gas and oil deposits in hydrodynamic traps. Higher Education Messenger: Oil and Gas, Moscow. 1984;11:3-6.

[8] Gutnikov AI, Zholdasov A, Zakirov SN. Nonstationary displacements of an oil deposit in a stream of stratal water. Messenger of Academy of Sciences of the USSR, Moscow. 1985;2:177-179.

[9] Zhirov VV. New methods for forecasting the composition of extracted production in the development of natural gas deposits. $\mathrm{PhD}$ Thesis. Moscow: VNII Gas, 1985.

[10] Shazhdekeeva NK, Mukhambetzhanov ST. On the properties of the solution of a certain problem of the theory of filtration with respect to phase transitions. Materials of the $\mathrm{V}$ international conference "Mathematical modeling and information technologies in education and science", dedicated to the 25th anniversary of computer science in the school. Almaty, 2010, pp. 114-118.

[11] Shazhdekeeva NK, Kammatov K, Adieva AA. On the existence of stationary solutions of a certain type of quasinelinear systems. Messenger of ASU, Atyrau. 2005;2:31-36.

[12] Frolov VV. Uniqueness theorems for the solution of the inverse heat conduction problem. Physics Engineering Journal. 1975;29(1):145-150. 